\title{
The role of model validation for assessing the size of the unmodeled dynamics
}

\author{
Lennart $\mathrm{LJUNG}^{+}$and Lei $\mathrm{GUO}^{++}$
}

October 9, 1995

\begin{abstract}
The problem of assessing the quality of a given, or estimated model is a central issue in system identification. Various new techniques for estimating bias and variance contributions to the model error have been suggested in the recent literature. In this contribution, classical model validation procedures are placed at the focus of our attention. We discuss the principles by which we reach confidence in a model through such validation techniques, and also how the distance to a "true" description can be estimated this way. In particular, we stress how the typical model validation procedure gives a direct measure of the model error of the model test, without referring to its ensemble properties. Several model error bounds are developed for various assumptions about the disturbances entering the system.
\end{abstract}

+ Department of Electrical Engineering, Linköping University, Linköping, S-58183, Sweden. Email: Ljung@isy.liu.se, Fax: (+46)13 282622.

++ Institute of Systems Science, Chinese Academy of Sciences, Beijing, 100080, P.R.China. Email: Lguo@iss03.iss.ac.cn, Fax: (86-10)2587343. 


\section{Introduction}

Model validation has always played a major role in System Identification, as a basic instrument for model structure selection and as the last "quality control" station before a model is delivered to the user [9], [14].

Methods for robust control design have pointed to the need for reliable model error bounds, for linear models preferably described as bounds on the frequency functions. A large number of approaches have been developed for this. See, e.g., [7],[8],[4],[3],[15]. For recent work on model validation in a worst-case context see [11] and [13]. Many of the contributions use deterministic frameworks to describe the noise and disturbances appearing in the system in order to avoid probabilistic, "soft", bounds. Approaches like "unknown-but-bounded" noises (the disturbances are assumed to be bounded, but no other assumptions are invoked), see e.g. [12], lead to setmembership procedures, which determine all models that are consistent with the noise bound given, see, e.g. [1], [10], [16].

In this contribution we shall take a different perspective. We place Model Validation in focus and try to interpret several identification concepts and approaches, as well as model quality aspects through the "eyes" of model validation.

We place ourselves in the following situation. A model is given. Let it be denoted by $\hat{G}$ (more specific notation will follow later). We are also given a data set $Z^{N}$ consisting of measured input-output data from a system. We do not know, or do not care, how the model was estimated, or constructed or given. We might not even know if the data set was used to construct the model. (However, some issues will turn out to depend on this fact.)

Our problem is to figure out if the model $\hat{G}$ is any good at describing the measured data, and perhaps also to give a statement how "far away" the model might be from a true description. We would like to approach this problem as naked as possible, and strip off common covers, such as "prior assumptions", "probabilistic frameworks", "worst case model properties" and the like. What are we then left with?

Well, a natural start is to consider the model's simulated response to the measured input signal. Let that simulated output be denoted by $\hat{y}$. We would then compare this model output with the actual measured output and contemplate how good the fit is. This is indeed common practice, and is perhaps the most useful, pragmatic way to gain confidence in (or reject) a model. This will be the starting point of our discussion. 
We shall first, in Section 2, discuss some typical statistics around the measured and simulated outputs. Note that "statistics" here means some bulk, numerical descriptions of the fit; this has nothing to do with probability theory. In particular, we shall discuss conventional Residual Analysis in this framework.

Section 3 gives the main theorem: A connection of algebraic nature between the model error, the input signal, the model validation test quantity and a noise/correlation term. The basic unknown quantity in this expression is the noise/correlation term. In Sections 4 and 5 the size of this terms is estimated in a deterministic and a stochastic framework, respectively.

\section{Some Notations}

We shall use the following notation. The input will be denoted by $u(t)$ and the output by $y(t)$. The data record thus is

$$
Z^{N}=\{y(1), u(1), \ldots, y(N), u(N)\}
$$

The input sequence $\{u(t), t=1, \ldots, N\}$ will throughout this paper be considered as a deterministic sequence, unless otherwise stated. We denote its periodogram by

$$
\left|U_{N}(\omega)\right|^{2}=\frac{1}{N}\left|\sum_{t=1}^{N} u(t) e^{-i \omega t}\right|^{2}
$$

The given model $\hat{G}$ will be assumed to be linear, and a function of the shift operator $q$ in the usual way: $\hat{G}(q)$. The simulated output will thus be

$$
\hat{y}(t)=\hat{G}(q) u(t)
$$

It may be that the model contains a noise assumption, typically in the form of an additive noise or disturbance $v(t)$ with certain properties. It would then be assumed that the actual output is generated as

$$
y_{m}(t)=\hat{G}(q) u(t)+v(t)
$$

(We append a subscript $m$ to stress the difference with the measured output.) The model could contain some "prejudice" about the properties of $v(t)$, but this is not at all essential to our discussion. A typical, conventional assumption would be that $v(t)$ is generated from a white noise source through a linear filter:

$$
v(t)=\hat{H}(q) e(t)
$$


Most of the model validation tests are based on simply the difference between the simulated and measured output:

$$
\varepsilon(t)=y(t)-\hat{y}(t)=y(t)-\hat{G}(q) u(t)
$$

For added generality, we shall consider possibly prefiltered model errors:

$$
\varepsilon(t)=L(q)[y(t)-\hat{y}(t)]=L(q)[y(t)-\hat{G}(q) u(t)]
$$

For example, if the model comes with a noise model (5), then a common choice of prefilter is $L(q)=\hat{H}^{-1}(q)$, since this would make $\varepsilon(t)$ equal to the model's prediction errors. This choice of prefilter is however not at all essential to our discussion.

In any case we shall call $\varepsilon(t)$ the Model Residuals ("model leftovers").

\section{Model Validation: Statistics Around the Residuals}

Typical model validation tests amount to computing the model residuals and giving some statistics about them. Note that this as such has nothing to do with probability theory. (It is another matter that statistical model validation often is complemented with probability theory and model assumptions to make probabilistic statements based on the residual statistics. See, e.g., [2].)

The following statistics for the model residuals are often used:

- The maximal absolute value of the residuals

$$
M_{N}^{\varepsilon}=\max _{1 \leq t \leq N}|\varepsilon(t)|
$$

- Mean, Variance and Mean Square of the residuals

$$
\begin{aligned}
m_{N}^{\varepsilon} & =\frac{1}{N} \sum_{t=1}^{N} \varepsilon(t) \\
V_{N}^{\varepsilon} & =\frac{1}{N} \sum_{t=1}^{N}\left(\varepsilon(t)-m_{N}^{\varepsilon}\right)^{2} \\
S_{N}^{\varepsilon} & =\frac{1}{N} \sum_{t=1}^{N} \varepsilon(t)^{2}=\left(m_{N}^{\varepsilon}\right)^{2}+V_{N}^{\varepsilon}
\end{aligned}
$$


- Correlation between residuals and past inputs.

Let

$$
\varphi(t)=[u(t), u(t-1), \ldots, u(t-M+1)]^{T}
$$

and

$$
R_{N}=\frac{1}{N} \sum_{t=1}^{N} \varphi(t) \varphi(t)^{T}
$$

Now form the following scalar measure of the correlation between past inputs (i.e. the vector $\varphi$ ) and the residuals:

$$
\tilde{\xi}_{N}^{M}=\frac{1}{N}\left\|\sum_{t=1}^{N} \varphi(t) \varepsilon(t)\right\|_{R_{N}^{-1}}^{2}
$$

Note that this quantity also can be written as

$$
\tilde{\xi}_{N}^{M}=\hat{r}_{\varepsilon u}^{T} R_{N}^{-1} \hat{r}_{\varepsilon u}
$$

where

$$
\hat{r}_{\varepsilon u}=\left[\hat{r}_{\varepsilon u}(0), \ldots, \hat{r}_{\varepsilon u}(M-1)\right]^{T}
$$

with

$$
\hat{r}_{\varepsilon u}(\tau)=\frac{1}{\sqrt{N}} \sum_{t=1}^{N} \varepsilon(t) u(t-\tau)
$$

Now, if we were prepared to introduce assumptions about the true system (the measured data $Z^{N}$ ), we could use the above statistical measures to make statements about the relationship between the model and the true system, typically using a probabilistic framework.

If we do not introduce any explicit assumptions about the true system, what is then the value of the statistics (8)-(14)? Well, we are essentially left only with induction. That is to say, we take the measures as indications of how the model will behave also in the future:

"Here is a model. On past data it has never produced a model error larger than 0.5. This indicates that in future data and future applications the error will also be below that value."

This type of induction has a strong intuitive appeal. 
In essence, this is the step that motivates the "unknown-but-bounded approach". Then a model or a set of models is sought that allows the preceeding statement with the smallest possible bound, or perhaps a physically reasonable bound.

Note, however, that the induction step is not at all tied to the unknownbut-bounded approach. Suppose we instead select the measure $S_{N}^{\varepsilon}$ as our primary statistics for describing the model error size. Then the Least Squares (Maximum Likelihood/Prediction Error) identification method emerges as a way to come up with a model that allows the "strongest" possible statement about past behavior.

How reliable is the induction step? It is clear that some sort of invariance assumption is behind all induction. To have some confidence in the induced statement about the future behavior of the model, we thus have to assume that certain things do not change. To look into the invariance of the behavior of $\varepsilon$ it is quite useful to reason as follows. (This will bring out the importance of the statistics (14)).

It is very useful to consider two sources for the model residual $\varepsilon$ : One source that originates from the input $u(t)$ and one that doesn't. With the (bold) assumption that these two sources are additive and the one that originates from the input is linear, we could write

$$
\varepsilon(t)=\tilde{G}(q) u(t)+v(t)
$$

Note that the distinction between the contributions to $\varepsilon$ is fundamental and has nothing to to with any probabilistic framework. We have not said anything about $v(t)$, except that it would not change, if we changed the input $u(t)$. We refer to (18) as the separation of the model residuals into Model Error and Disturbances.

The division (18) shows one weakness with induction for measures like $M_{N}^{\varepsilon}$ and $S_{N}^{\varepsilon}$ going from one data set to another. The implicit invariance assumption about the properties of $\varepsilon$ would require both the input $u$ and the disturbances $v$ to have invariant properties in the two sets. Only if we would have indications that $\tilde{G}$ is of insignificant size, we could allow inductions from one data set to another with different types of input properties. The purpose of the statistics $\tilde{\xi}_{N}^{M}$ in (14) is exactly to assess the size of $\tilde{G}$. We shall see this clearly in Section 3. (One might add that more sophisticated statistics will be required to assess more complicated contributions from $u$ to $\varepsilon$ ).

In any case, it is clear that the induction about the size of the model residuals from one data set to another is much more reasonable if the statistics 
$\tilde{\xi}_{N}^{M}$ has given a small value ("small" must be evaluated in comparison with $S_{N}^{\varepsilon}$ in (11)).

We might add that the assumption (18) is equivalent to assuming that the data $Z^{N}$ have been generated by a "true system"

$$
y(t)=G_{0}(q) u(t)+v(t)
$$

where

$$
\tilde{G}(q)=G_{0}(q)-\hat{G}(q)
$$

\section{The Main Theorem}

The question now is, what can be said about the model error $\tilde{G}$ based on the information in $Z^{N}$.

The procedure will be to apply the residual analysis of Section 2. Form

$$
\varepsilon(t)=L(q)(y(t)-\hat{G}(q) u(t))
$$

and then $\tilde{\xi}_{N}^{M}$ as in (12)-(14). In these calculations replace $u(t)$ outside the interval $[1, N]$ by zero. Then our main technical result is the following theorem:

Theorem 3.1 Assume that the data set $Z^{N}$ in (1) is subject to (19). Let $\hat{G}$ be a given model, and let $\tilde{\xi}_{N}^{M}$ be formed from the data by (12) - (14). Assume that $R_{N}>\delta I$. Then the model error (20) obeys

$$
\begin{aligned}
& {\left[\frac{1}{2 \pi} \int_{-\pi}^{\pi}\left|\tilde{G}\left(e^{i \omega}\right)\right|^{2}\left|L\left(e^{i \omega}\right)\right|^{2}\left|U_{N}(\omega)\right|^{2} d \omega\right]^{1 / 2} \leq } \\
\leq & (1+\eta)\left[\frac{1}{N} \tilde{\xi}_{N}^{M}\right]^{1 / 2}+(1+\eta) x_{N}+(2+\eta) C_{u} \sum_{k=M}^{\infty}\left|\rho_{k}\right|
\end{aligned}
$$

Here

- $x_{N}=\left\|\frac{1}{N} \sum_{t=1}^{N} \tilde{v}(t) \varphi(t)\right\|_{R_{N}^{-1}}$

- $\tilde{v}(t)=L(q) v(t)$

- $\rho_{k}$ is the impulse response of $L(q) \tilde{G}(q)$

- $\left|U_{N}\right|^{2}$ is the periodogram (2). 
- $\eta=\frac{C_{u} M}{\sqrt{N \delta}}$

- $C_{u}=\max _{1 \leq t \leq N}|u(t)|$.

If the input is tapered so that $u(t)=0$ for $t=N-M+1, \ldots N$, the number $\eta$ can be taken as zero.

Proof. See Appendix A.

Let us make a number of comments:

- The theorem is really just a statement about the relationship between the sequences $\tilde{v}(t)=L(q)\left[y(t)-G_{0}(q) u(t)\right]$, and $\varepsilon(t)=L(q)[y(t)-$ $\hat{G}(q) u(t)]$ on the one hand and the given transfer functions $L(q), G_{0}(q), \hat{G}(q)$ together with the given sequences $u(t), y(t)$ on the other hand. There are as yet no stochastic assumptions whatsoever, and no requirement that the "model" $\hat{G}$ may or may not be constructed from the given data.

- By the choice of prefilter $L(q)$ we can probe the size of the model error over arbitrarily small frequency intervals. However, by making this filter very narrow band, we will also typically increase the size of the impulse response tail. (Narrow band filters have slowly decaying impulse responses.)

- Note also that if $u$ and $y$ are subject to $y(t)=G_{0}(q) u(t)+v(t)$, then $u_{F}(t)=L(q) u(t)$ and $y_{F}(t)=L(q) y(t)$ will be subject to $y_{F}=$ $G_{0}(q) u_{F}(t)+v_{F}(t)$, where $v_{F}(t)=L(q) v(t)$. This means that the theorem could also be applied directly using the sequences $u_{F}$ and $y_{F}$, giving an alternative bound on the model error, which could be both stronger and weaker than the one obtained with the filter $L$ explicitly present as in the Theorem 3.1.

- If the model $\hat{G}(q)$ has been estimated as an $M$ :th order FIR model from the data set $Z^{N}$ using the least squares method, then by construction $\tilde{\xi}_{N}^{M}=0$.

- For the quantities on the right hand side, we note that $\tilde{\xi}_{N}^{M}$ is known by the user, as well as $\eta, N$ and $C_{u}$. The tail of the impulse response $\rho_{k}$ beyond lag $M$ is typically not known. It is an unavoidable term, since no such lag has been tested. The size of this term has to be dealt with by prior assumptions. 
- The only essential unknown term is $x_{N}$. We shall call this "The correlation term". The size and the bounds on this term will relate to noise assumptions and we will deal with these in some detail in the two following sections.

\section{Non-probabilistic Bounds on the Correla- tion Term}

The term $x_{N}$ measures the correlation between the input $u$ and the filtered disturbance $\tilde{v}$. In a deterministic setting it is not so easy to formalize what we should mean by "uncorrelated disturbances". One could of course postulate that the disturbance sequence that we expect to enter the process is such that quantities like $x_{N}$ decay like $1 / N$ or $\log N / N$ or in any other way. That would give us hard model error bounds in Theorem 3.1.

We shall instead consider two other bounds on $x_{N}$ that stem from less complex assumptions, one that is always valid and one that is valid for periodic inputs.

\subsection{A simple bound}

Suppose that $\tilde{v}(t)$ is any sequence, and all that is known about it is an amplitude or an energy bound. By Lemma B.1

$$
x_{N} \leq\left[\frac{1}{N} \sum_{t=1}^{N} \tilde{v}^{2}(t)\right]^{1 / 2}
$$

A bound on disturbance power or amplitude will thus directly give a hard model error bound in (21).

Combining it with FIR-modeling, the result can then be used in Theorem 3.1 to yield, for example, the following explicit result:

Theorem 4.1 Assume that a tapered and bounded input $u(t), t=1, \ldots, N$ (the trailing $M$ values of $u(t)$ are zero, and $|u(t)| \leq C_{u}$ ) has been applied to the system

$$
y(t)=G_{0}(q) u(t)+v(t)
$$


where $|v(t)| \leq C_{v}$. Let $\hat{G}_{N}(q)$ be constructed from the data as an Mth order FIR (finite impulse response) model, using the least squares method. Then

$$
\begin{aligned}
& {\left[\frac{1}{2 \pi} \int_{-\pi}^{\pi}\left|G_{0}\left(e^{i \omega}\right)-\hat{G}_{N}\left(e^{i \omega}\right)\right|^{2}\left|U_{N}(\omega)\right|^{2} d \omega\right]^{1 / 2} \leq } \\
\leq & C_{v}+2 C_{u} \sum_{k=M}^{\infty}\left|\rho_{k}\right|
\end{aligned}
$$

where $\rho_{k}$ is the impulse response of $G_{0}(q)$, and $\left|U_{N}(\omega)\right|^{2}$ is the periodogram of the input.

Remark: A lower bound on the matrix $R_{N}$ is not required for this result.

\subsection{The Case of Periodic Input}

It is of course desirable that the quantity $x_{N}$ should be as small as possible. We noted that the variable is a measure of the "correlation" between the noise sequence $\tilde{v}(t)$ and the input $u(t)$. The Schwarz' type inequality (22) is the most conservative bound allowing all kinds of relationships between the two signals. In fact, as always, equality in this bound is achieved when the two signals are exactly "parallel". To get beyond this bound we thus have to invoke some kind of independence properties between $\tilde{v}(t)$ and $u(t)$. As soon as we introduce a stochastic framework for the signals, this is easy and "classical" to achieve, but even in a deterministic perspective some things can be obtained. One way is to assume that the input is periodic, and that the noise term isn't. We have the following result:

Lemma 4.1 Under the notation of Theorem 3.1, assume that the input is periodic with period $P$, then the following bound holds:

$$
x_{N} \leq C_{N} \cdot \max _{\omega}|\tilde{V}(\omega)|
$$

where $\tilde{V}(\omega)$ is the discrete time Fourier transform of $\tilde{v}(t)$,

$$
\tilde{V}(\omega)=\frac{1}{\sqrt{N}} \sum_{t=1}^{N} \tilde{v}(t) e^{-i t \omega}
$$

and

$$
C_{N}=\frac{1+\log (N / P+1)}{\sqrt{N}} \cdot \frac{M P}{\sqrt{\delta}} \cdot C_{u}
$$


The proof is given in Appendix B. Note that the requirement that $R_{N}>$ $\delta I$ effectively means that $P>M$.

The lemma says that for periodic input and for noises with suitably smooth spectrum, the model error decays like $O\left(\frac{1}{\sqrt{N}}\right)$, save that a factor with logarithmatic order of increasing is neglected. This is essentially the same result that is obtained in the classical stochastic framework (see below).

With this lemma used in Theorem 3.1 the only remaining unknown quantity is the noise periodogram $|\tilde{V}(\omega)|^{2}$. It should also be noted that the typical model validation companion test, the one that checks the whiteness of the residuals, indeed is a way to evaluate this quantity and also secure the flatness of the spectrum of $\tilde{v}$. This test consequently links in with an attempt to quantify the total model error according to Theorem 3.1 and the lemma above.

There is a close link between Theorem 3.1, the above lemma and some basic results about the Empirical Transfer Function Estimate (ETFE). In, e.g., [9], Section 6.3, it is shown that

$$
\hat{\hat{G}}_{N}\left(e^{i \omega}\right)-G_{0}\left(e^{i \omega}\right)=\frac{V_{N}(\omega)}{U_{N}(\omega)}+\text { vanishing term for periodic input }
$$

Here $U_{N}$ and $V_{N}$ are the Fourier transforms of the input and the noise and the ETFE $\hat{G}$ is the ratio between the output and input Fourier transform. For a periodic input and a non-periodic noise, the error thus decays like $1 / \sqrt{N}$ at those frequencies present in the input.

\section{Probabilistic bounds on the correlation term}

The division of the model residual (18) into a model error part and a disturbance part clearly manifests that the disturbance $v$ should have nothing to do with the input. To formalize this notion, it is customary to introduce a probabilistic framework and assume that $u$ and $v$ are mutually independent sequences of random variables. It might be stressed that it is this independence assumption that is the essential contribution of the probabilistic framework and that gives the basic model properties.

If the model $\hat{G}$ is obtained from a data set, independent of $Z^{N}$, then the below results will give direct probabilistic bounds on the given model's error. If the model has been estimated from $Z^{N}$ the results are more difficult to interpret, since there will be correlation between the terms $\tilde{G}$ and $x_{N}$. 


\subsection{Direct Probabilistic Bounds on $x_{N}$}

We shall in this subsection assume that $v(t)$ is a stationary stochastic process with zero mean, independent of the input (which we anyway treat as a deterministic sequence). The covariance function of $v(t)$ is assumed to decay so fast that the spectrum is defined. It will be denoted by $\Phi_{v}(\omega)$. Under these assumptions, $x_{N}$ becomes a random variable. According to Lemma B.1

$$
\mathrm{E} x_{N}^{2} \leq \frac{M}{N} \max _{\omega}\left|L\left(e^{i \omega}\right)\right|^{2} \Phi_{v}(\omega)
$$

Moreover, under weak assumptions the central limit theorem can be applied to show that

$$
R_{N}^{-1 / 2} \frac{1}{\sqrt{N}} \sum_{t=1}^{N} \varphi(t) \tilde{v}(t)
$$

converges in distribution to the Normal distribution with zero mean and covariance matrix $S$, where, according to (29)

$$
\operatorname{tr} S=\lim _{N \rightarrow \infty} \operatorname{E} N x_{N}^{2} \leq P_{x}
$$

where

$$
P_{x}=M \max _{\omega}\left|L\left(e^{i \omega}\right)\right|^{2} \Phi_{v}(\omega)
$$

We can from this develop direct probabilistic bounds for $x_{N}$ in (21) and hence for the model error. A conservative bound is, e.g., obtained from the implication

$$
x_{N}>\epsilon \Rightarrow\left(\beta_{N}^{(i)}\right)^{2}>\frac{N}{M} \epsilon^{2} \text { for some } i
$$

(subscript $(i)$ denotes the $i$ :th component). Since $\left(\beta_{N}^{(i)}\right)^{2}$ is asymptotically $\chi^{2}$ distributed with one degree of freedom we find that

$$
P\left(x_{N}>\epsilon\right) \leq M \chi_{1}\left(\frac{N \epsilon^{2}}{M P_{x}}\right)
$$

where the last symbol is the probability that a $\chi^{2}$-distributed random variable with one degree of freedom is larger than the indicated argument and $P_{x}$ is defined by (31). 


\subsection{Bounds with Probability One}

Let us assume that either

(i) $\{v(t)\}$ is bounded deterministic and $\{u(t)\}$ is a zero mean stationary ARMA process; or

(ii) $\{u(t)\}$ is bounded deterministic and $\{v(t)\}$ is a zero mean stationary ARMA process.

Then, the correlation term $x_{N}$ in Theorem 3.1 can be estimated by (see, e.g., [5])

$$
x_{N}^{2} \leq C_{x} \frac{1}{N} \log \log N \quad \text { a.s. }
$$

for a random variable $C_{x}$ with bounded variance. Together with some assumption about the decay of the tail of the impulse response, and the measured value of $\tilde{\xi}_{N}^{M}$ this bound gives quite an explicit bound for the model error in (21).

Note that, as indicated by (33), a bounded deterministic disturbance $\{v(t)\}$ does not influence the asymptotic error bound, as long as the input sequence $\{u(t)\}$ is suitably chosen (see also, [5] and [6]). This essentially improves the related error bounds derived in the existing deterministic framework of system identification.

\subsection{Hard Bound if the Model Validation Test Passes}

We can twist the probabilistic bounds of section 5.1 around to give hard bounds on the model error, in case the model validation test passes with a certain probability.

Let us first describe the typical model validation test.If $\varepsilon$ is white noise and independent of $u$, then $\hat{r}_{\varepsilon u}$, defined by (16) will be asymptotically normal with covariance matrix $R_{N} \cdot \mathrm{E} \varepsilon^{2}(t)$. Hence the test variable

$$
\begin{array}{r}
\xi_{N}^{M}=\frac{1}{\hat{r}_{\varepsilon}(0)} \tilde{\xi}_{N}^{M} \\
\hat{r}_{\varepsilon}(0)=\frac{1}{N} \sum_{t=1}^{N} \varepsilon^{2}(t)
\end{array}
$$

will be asymptotically $\chi^{2}$-distributed with $M$ degrees of freedom:

$$
\xi_{N}^{M} \sim \chi^{2}(M)
$$


The validation procedure is thus to form (34) and check its size in $\chi^{2}$ confidence level tables.

Now, if, in a given case $\xi_{N}^{M}$ turns out to be less than a chosen test level $\chi_{\alpha}$, we say that the hypothesis that $u$ and $\varepsilon$ are uncorrelated can be accepted there is no conclusive evidence against it. If such a test passes with a certain probability, we can draw some conclusions about the actual model error. To develop such a result, we first prove the following lemma:

Lemma 5.1 Suppose

$$
A \leq B \cdot \xi+C+D
$$

where $B, \xi$ and $C$ are random variables and $A, D$ are deterministic quantities. Suppose that

$$
P\left(\xi \leq C_{\xi}\right) \geq \alpha
$$

$(P(\Gamma)$ denotes the probability of the event $\Gamma)$. Then

$$
A \leq \sqrt{\frac{\mathrm{E} B^{2}}{\alpha}} C_{\xi}+\sqrt{\frac{\mathrm{E} C^{2}}{\alpha}}+D
$$

Proof: Multiply (37) with the indicator function $I_{H}$ for the event $H=\{\xi \leq$ $\left.C_{\xi}\right\}$ :

$$
A I_{H} \leq B I_{H} C_{\xi}+C I_{H}+D I_{H}
$$

Then take expectation and use Schwarz' inequality:

$$
\begin{aligned}
A P(H) & \leq C_{\xi} \mathrm{E}\left(B I_{H}\right)+\mathrm{E}\left(C I_{H}\right)+D P(H) \\
& \leq C_{\xi} \sqrt{\mathrm{E} B^{2} \cdot \mathrm{E} I_{H}^{2}}+\sqrt{\mathrm{E} C^{2} \cdot \mathrm{E} I_{H}^{2}}+D P(H) \\
& =C_{\xi} \sqrt{P(H) \cdot \mathrm{E} B^{2}}+\sqrt{P(H) \cdot \mathrm{E} C^{2}}+D P(H)
\end{aligned}
$$

which proves the result.

Based on this result and on Lemma B.1 we can formulate Theorem 3.1 as follows:

Theorem 5.1 Assume that the data set $Z^{N}$ in (1) is subject to (19). Assume the input $u$ is deterministic and that $v(t)$ is a stationary stochastic process with spectrum $\Phi_{v}(\omega)$. Let $\hat{G}$ be a given model, independent of $Z^{N}$ and let the test quantity $\xi_{N}^{M}$ be formed from the data by (12) - (14), (34). Assume that 
the probability that the test $\xi_{N}^{M} \leq \chi_{1}$ passes is at least $\alpha$, and that $R_{N}>\delta I$. Then the model error (20) obeys

$$
\begin{aligned}
& {\left[\frac{1}{2 \pi} \int_{-\pi}^{\pi}\left|\tilde{G}\left(e^{i \omega}\right)\right|^{2}\left|L\left(e^{i \omega}\right) U_{N}(\omega)\right|^{2} d \omega\right]^{1 / 2} \leq } \\
\leq & \frac{1+\eta}{\sqrt{N \alpha}}\left[\left[\chi_{1}\right]^{1 / 2}\left[\operatorname{Er}_{\varepsilon}(0)\right]^{1 / 2}+\max _{\omega}\left[M\left|L\left(e^{i \omega}\right)\right|^{2} \Phi_{v}(\omega)\right]^{1 / 2}\right] \\
+ & (2+\eta) C_{u} \sum_{k=M}^{\infty}\left|\rho_{k}\right|
\end{aligned}
$$

Here $\left|U_{N}\right|^{2}, \eta, \rho_{k}$ and $C_{u}$ are defined as in Theorem 3.1, and the same comment about $\eta$ and input tapering applies here also.

Proof: Apply Lemma 5.1 to (21). Write $\xi_{N}^{M}$ as $\hat{r}_{\varepsilon}(0) \xi_{N}^{M}$ and identify $\hat{r}_{\varepsilon}(0)$ with $B$ and $\sqrt{\xi_{N}^{M}}$ with $\xi$ in the lemma.

Note that (40) is a hard bound statement about the error of the (given or estimated) model $\hat{G}$. It is true that we have to assume that the test passes with a certain probability, and this will of course not be easy to verify. By performing a number of validation tests on different data sets, we however get some insight in this probability.

\subsection{Model Mean Square Error Bounds}

So far we have only made statements about one particular, given model $\hat{G}$. If the model is given, and independent of the test data $Z^{N}$ (i.e. it has not been estimated from this data set), there is really no reason to look into any ensemble properties.

The situation is different if the model has been estimated using $Z^{N}$. It will then depend on the $v$-sequence. All inequalities still hold, and it is meaningful to take the expectation to look into the average properties of the model.

Some care has to be exercised regarding the tail term with the impulse responses of the model error. To deal with this we introduce the following procedure:

1. Assume that a prior bound is know for the tail of the impulse response of the true system $G_{0}(q)$ :

$$
\bar{\rho}_{M} \geq \sum_{t=M}^{\infty}\left|g_{0}(t)\right|
$$


2. Any produced model is projected so that the tail of the model's impulse response is also bounded as in (41).

3. Pick a test length $M$.

4. Pick a test level $C_{\xi}$ and estimate models in your favorite model classes (subject to step (2) above) from $Z^{N}$ until a model is found that passes the validation test

$$
\tilde{\xi}_{N}^{M} \leq C_{\xi}
$$

for the data $Z^{N}$. ( $\left(\tilde{\xi}_{N}^{M}\right.$ defined by (14).) This will always be possible to achieve, at least if the model classes contain finite impulse response models of length $M$, since that will give $\tilde{\xi}_{N}^{M}=0$.

For models found in this way we have the following result.

Theorem 5.2 Assume that the data set $Z^{N}$ in (1) is subject to (19), where the tail of the true impulse response is bounded as in (41). Let $\hat{G}$ be any model estimated from the data according to the procedure outlined above. Then the model's mean square error obeys

$$
\begin{aligned}
& \frac{1}{2 \pi} \int_{-\pi}^{\pi} \mathrm{E}\left|\tilde{G}\left(e^{i \omega}\right)\right|^{2}\left|U_{N}(\omega)\right|^{2} d \omega \leq \\
\leq & \frac{3(1+\eta)^{2}}{N}\left[C_{\xi}+M \max _{\omega} \Phi_{v}(\omega)\right]+12(2+\eta)^{2} C_{u}^{2} \bar{\rho}_{M}^{2}
\end{aligned}
$$

Here $\eta, C_{u}$ and $|U|^{2}$ are defined as in Theorem 3.1, while $C_{\xi}$ is the test limit for the validation and $\bar{\rho}_{M}$ is defined by (41), while $\Phi_{v}$ is the spectrum of $v$.

Proof: Square (21) and take expectation. Use Lemma B.1 for the $x_{N}$ term and that the impulse response of the model error is less than the sum of the responses of the true system and the model.

The theorem tells as that the model mean square error decreases as $M / N$ plus the tail of the impulse response. The best bound is obtained by choosing $M$ so that $M / N \sim \bar{\rho}_{M}$. For an exponentially stable system $\bar{\rho}_{M} \sim \lambda^{M}$, which means that $M$ should be chosen as $\sim \log N$. That gives a total mean square error bound that decays like $\log N / N$. 


\section{Conclusions}

In this paper we have studied what a typical model validation test implies in terms of the model error, expressed in the frequency domain. There has been a considerable interest lately in quantifying model quality in terms of bounds on its frequency function, e.g. [7],[8],[4][3],[15].

A substantial part of that literature discusses the error in terms of bias and variance contributions. The idea then is that the variance terms is easily handled using "classical" probabilistic estimation theory, while the bias estimation part is much harder.

We have taken a somewhat different perspective in the main result, Theorem 3.1, showing that a traditional model validation test immediately gives a "hard" bound on an integral of the frequency domain model error for the actual model under test. There is consequently no real need to look into the ensemble properties. Indeed, it is much more natural to have a statement directly about the actual model we are working with.

It is true that the hard bound depends on some prior information (the decay of the true impulse response function) and a term $\left(x_{N}\right)$ that reflects knowledge/assumptions about the noise in the true system. We have shown how the latter term can be bounded, with hard bounds, in probability and in mean square under various noise assumption, including purely deterministic ones.

The model error bound does not come pointwise in the frequency domain. In fact, based on $N$ data and $M$-lag tests, we can penetrate the frequency domain only up to a certain resolution. This is manifested as the bound on integrated versions of the model error. The user has a certain amount of freedom to focus on narrow frequency regions using the prefilter $L$. For given $N$ and $M$ these regions cannot, however be made arbitrarily narrow, since the tail term (last term in(21)) would increase as the pass band of $L$ narrows. This, again, is unavoidable, in the light of the uncertainty principle between frequency resolution and data record length.

\section{A Proof of Theorem 3.1}

\section{A.1 Some Preliminaries}

We shall use the following lemma. 
Lemma A.1 Let

$$
\begin{gathered}
w(t)=N(q) u(t)=\sum_{k=0}^{\infty} n_{k} u(t-k) \\
\varphi(t)=\left[u(t), \ldots, u(t-M+1) ; R_{N}=\frac{1}{N} \sum_{t=1}^{N} \varphi(t) \varphi^{T}(t)\right. \\
U_{N}(\omega)=\frac{1}{\sqrt{N}} \sum_{t=1}^{N} u(t) e^{-i \omega t}
\end{gathered}
$$

Assume that

$$
\begin{gathered}
|u(t)| \leq C_{u} \quad t=1, \ldots, N \\
R_{N}>\delta I
\end{gathered}
$$

Let

$$
\tilde{\rho}_{M}=\sum_{k=M}^{\infty}\left|n_{k}\right|
$$

and define

$$
\beta^{2}=\left\|\frac{1}{N} \sum_{t=1}^{N} w(t) \varphi(t)\right\|_{R_{N}^{-1}}^{2} \quad \text { and } \quad B^{2}=\frac{1}{2 \pi} \int_{-\pi}^{\pi}\left|N\left(e^{i \omega}\right)\right|^{2}\left|U_{N}(\omega)\right|^{2} d \omega
$$

Then for $\eta=\frac{C_{u} M}{\sqrt{N \delta}}$

$$
B \leq(1+\eta) \beta+(2+\eta) C_{u} \tilde{\rho}_{M}
$$

If the input is tapered, so that $u(N-M+1) \ldots u(N)$ are all zero, then $\eta$ can be taken as zero.

The proof of the lemma will be given at the end of this appendix.

\section{A.2 Proof of the Theorem}

Let

$$
\tilde{G}(q)=G_{0}(q)-\hat{G}(q)
$$

and

$$
\begin{gathered}
\tilde{u}(t)=L(q) \tilde{G}(q) u(t) \\
\tilde{v}(t)=L(q) v(t)
\end{gathered}
$$


Then

$$
\varepsilon(t)=\tilde{u}(t)+\tilde{v}(t)
$$

Thus

$$
\begin{aligned}
{\left[\frac{1}{N} \tilde{\xi}_{N}^{M}\right]^{1 / 2} } & =\left\|\frac{1}{N} \sum_{t=1}^{N} \varepsilon(t) \varphi(t)\right\|_{R_{N}^{-1}}=\left\|\frac{1}{N} \sum_{t=1}^{N}(\tilde{u}(t)+\tilde{v}(t)) \varphi(t)\right\|_{R_{N}^{-1}} \\
& \geq \gamma-x
\end{aligned}
$$

where

$$
\gamma=\left\|\frac{1}{N} \sum_{t=1}^{N} \tilde{u}(t) \varphi(t)\right\|_{R_{N}^{-1}}
$$

and $x$ is defined in the theorem.

By Lemma A.1 we then have that

$$
\left[\frac{1}{2 \pi} \int_{-\pi}^{\pi}\left|\tilde{G}\left(e^{-i \omega}\right)\right|^{2}\left|L\left(e^{i \omega}\right) U(\omega)\right|^{2} d \omega\right]^{1 / 2} \leq(1+\eta) \gamma+(2+\eta) C_{u} \tilde{\rho}_{M}
$$

which, together with (49) proves the theorem.

\section{A.3 Proof of Lemma A.1}

Denote

$$
n=\left[\begin{array}{llll}
n_{0} & n_{1} \ldots & n_{M}
\end{array}\right]^{T}
$$

Then

$$
w(t)=n^{T} \varphi(t)+\sum_{k=M}^{\infty} n_{k} u(t-k)=n^{T} \varphi(t)+\tilde{w}(t)
$$

The second term is bounded by

$$
|\tilde{w}(t)|=\left|\sum_{k=M}^{\infty} n_{k} u(t-k)\right| \leq C_{w}
$$

under the assumptions of the lemma. Here, for convenience, we introduced the notation

$$
C_{w}=C_{u} \cdot \tilde{\rho}_{M}
$$


Inserting (50) into (46) gives

$$
\begin{aligned}
\beta & =\left\|\frac{1}{N} \sum_{t=1}^{N}\left[n^{T} \varphi(t) \varphi^{T}(t)+\tilde{w}(t) \varphi^{T}(t)\right]\right\|_{R_{N}^{-1}} \\
& =\left\|R_{N}^{1 / 2} n+R_{N}^{-1 / 2} \frac{1}{N} \sum_{t=1}^{N} \tilde{w}(t) \varphi(t)\right\| \\
& \geq\left\|R_{N}^{1 / 2} n\right\|-\left\|R_{N}^{-1 / 2} \frac{1}{N} \sum_{t=1}^{N} \tilde{w}(t) \varphi(t)\right\| \geq\left\|R_{N}^{1 / 2} n\right\|-C_{w}
\end{aligned}
$$

where for the last inequality we have used Lemma B.1 and (51).

Let us now turn to the quantity $n^{T} R_{N} n$. Define first

$$
\bar{u}(t)=\left\{\begin{array}{cc}
u(t) & \text { if } \quad 1 \leq t \leq N \\
0 & \text { else }
\end{array}\right.
$$

Then

$$
\begin{gathered}
\left|U_{N}(\omega)\right|^{2}=\frac{1}{N}\left|\sum_{t=1}^{N} u(t) e^{-i \omega t}\right|^{2}=\frac{1}{N}\left|\sum_{t=-\infty}^{\infty} \bar{u}(t) e^{-i \omega t}\right|^{2}= \\
=\frac{1}{N} \sum_{t=-\infty}^{\infty} \sum_{s=-\infty}^{\infty} \bar{u}(t) \bar{u}(s) e^{-i \omega(t-s)}=\sum_{\tau=-\infty}^{\infty} \frac{1}{N} \sum_{t=-\infty}^{\infty} \bar{u}(t) \bar{u}(t-\tau) e^{-i \omega \tau}
\end{gathered}
$$

Let

$$
\hat{r}(\tau)=\frac{1}{N} \sum_{t=-\infty}^{\infty} \bar{u}(t) \bar{u}(t-\tau)
$$

so that

$$
\hat{r}(k-\ell)=\frac{1}{2 \pi} \int_{-\pi}^{\pi}\left|U_{N}(\omega)\right|^{2} e^{+i \omega(k-\ell)} d \omega .
$$

The $(k, \ell)$ element of $R_{N}$ is

$$
R_{N}^{(k, \ell)}=\frac{1}{N} \sum_{t=\max (k, \ell)}^{N} u(t-k+1) u(t-\ell+1)
$$

SO

$$
\left|R_{N}^{(k, \ell)}-\hat{r}(k-\ell)\right| \leq C_{u}^{2} \cdot \max (k, \ell) / N \leq C_{u}^{2} M / N
$$

(Note:If the input is tapered as defined in the theorem, this bound is actually zero.) 

Then

Let $\hat{R}_{N}$ be the Toeplitz matrix built up from $\hat{r}(\tau)$ analogously to $R_{N}$.

$$
\left\|R_{N}-\hat{R}_{N}\right\| \leq C_{u}^{2} M^{2} / N
$$

This gives that

$$
\begin{aligned}
n^{T} R_{N} n & =\frac{1}{2 \pi} \int_{-\pi}^{\pi} \sum_{k, \ell=0}^{M-1} n_{k} n_{\ell} e^{i \omega(k-\ell)}\left|U_{N}(\omega)\right|^{2} d \omega+f_{1}= \\
& =\frac{1}{2 \pi} \int_{-\pi}^{\pi}\left|\sum_{k=0}^{M-1} n_{k} e^{-i \omega k}\right|^{2}\left|U_{N}(\omega)\right|^{2} d \omega+f_{1}
\end{aligned}
$$

where, using (55) and the condition $R_{N}>\delta I$.

$$
\|\left. f_{1}|=| n^{T}\left(R_{N}-\hat{R}_{N}\right) n\left|\leq \frac{C_{u}^{2} \cdot M^{2}}{N}\right| n\right|^{2} \leq \frac{c_{u}^{2} M^{2}}{N} n^{T} R_{N} n / \delta
$$

Let us define the $L_{2}$-norm of any complex function $F(\omega)$ on $[0,2 \pi]$ as

$$
\|F(\omega)\|_{2}=\left[\frac{1}{2 \pi} \int_{0}^{2 \pi}|F(\omega)|^{2} d \omega\right]^{1 / 2}
$$

By (56), (57), and the definition of $\eta$, we then have

$$
\begin{aligned}
\left|n^{T} R_{N}^{1 / 2}\right| & \geq\left\|\left(\sum_{k=0}^{M-1} n_{k} e^{-i \omega k}\right) U_{N}(\omega)\right\|_{2}-\left|f_{1}\right|^{1 / 2} \\
& \geq\left\|\left[N\left(e^{i \omega}\right)-\sum_{k=M}^{\infty} n_{k} e^{-i \omega k}\right] U_{N}(\omega)\right\|_{2}-\eta\left|n^{T} R_{N}^{1 / 2}\right| \\
& \geq B-\left\|\sum_{k=M}^{\infty} n_{k} e^{-i \omega k} U_{N}(\omega)\right\|_{2}-\eta\left|n^{T} R_{N}^{1 / 2}\right| \\
& \geq B-\tilde{\rho}_{M} C_{u}-\eta\left|n^{T} R_{N}^{1 / 2}\right|
\end{aligned}
$$

This implies that (by (52)),

$$
\left|n^{T} R_{N}^{1 / 2}\right| \geq \frac{1}{1+\eta}\left[B-C_{w}\right]
$$

Substituting this into (53), we have

$$
\beta \geq \frac{1}{1+\eta}\left[B-(2+\eta) C_{w}\right]
$$

which proves the desired result. 


\section{B Bounds on the correlation term}

We shall in this appendix study the term $x_{N}$ in Theorem 3.1 and develop a few bound for it. Consider the quantity

$$
x_{N}=\left\|\frac{1}{N} \sum_{t=1}^{N} w(t) \varphi(t)\right\|_{R_{N}^{-1}}=\left\|R_{N}^{-1 / 2} \frac{1}{N} \sum_{t=1}^{N} w(t) \varphi(t)\right\|
$$

where

$$
R_{N}=\frac{1}{N} \sum_{t=1}^{N} \varphi(t) \varphi^{T}(t)
$$

Assume that $\varphi(t)$ is a sequence of column vectors of length $M$. We have the following bounds on this quantity.

Lemma B.1 Let $x_{N}$ be defined by (62)-(63). Then

a $x_{N}^{2} \leq \frac{1}{N} \sum_{t=1}^{N}[w(t)]^{2}$

b Assume that $w(t)$ is a stationary random process with spectrum $\Phi_{w}(\omega)$, independent of $\varphi$. Then

$$
E x_{N}^{2} \leq \frac{M}{N} \max _{\omega} \Phi_{w}(\omega)
$$

Proof: Set

$$
W=[w(1), \ldots, w(N)]^{T} ; \quad \Psi=[\varphi(1), \ldots, \varphi(N)]^{T}
$$

Then

$$
x_{N}^{2}=\left|R_{N}^{-1 / 2} \frac{1}{N} \sum_{t=1}^{N} w(t) \varphi(t)\right|^{2}=\frac{1}{N} W^{T} \Psi^{T}\left(\Psi \Psi^{T}\right)^{-1} \Psi W
$$

Let $H=\Psi^{T}\left(\Psi \Psi^{T}\right)^{-1} \Psi$. Then $H^{2}=H$, so that the eigenvalues of $H$ are either 0 or 1 . Hence

$$
W^{T} H W \leq W^{T} W=\sum_{t=1}^{N}|w(t)|^{2}
$$

which proves claim a) of the lemma.

We now turn to claim b). By the properties of $H$ we know that there is an orthogonal matrix

$$
P=\left[p_{1}, \ldots, p_{N}\right], \quad p_{s} \in R^{N}
$$


such that

$$
H=P\left(\begin{array}{cc}
I_{M} & 0 \\
0 & 0
\end{array}\right) P^{T}=\left[p_{1}, \ldots, p_{M}\right]\left[p_{1}, \ldots, p_{M}\right]^{T}
$$

Hence

$$
W^{T} \Psi^{T}\left(\Psi \Psi^{T}\right)^{-1} \Psi W=\sum_{s=1}^{M}\left(W^{T} p_{s}\right)^{2}=\sum_{s=1}^{M}\left(\sum_{j=1}^{N} w(j) p_{s j}\right)^{2}
$$

where

$$
p_{s}=\left[p_{s 1}, \ldots, p_{s N}\right]^{T}
$$

Taking expectation and using the identity

$$
\mathrm{E} w(j) w(k)=\frac{1}{2 \pi} \int_{-\pi}^{\pi} \Phi_{w}(\omega) e^{-i \omega(k-j)} d \omega
$$

we have

$$
\begin{aligned}
\mathrm{E} W^{T} \Psi^{T}\left(\Psi \Psi^{T}\right)^{-1} \Psi W & =\frac{1}{2 \pi} \sum_{s=1}^{M} \int_{-\pi}^{\pi} \Phi_{w}(\omega) \sum_{j, k=1}^{N} p_{s j} p_{s k} e^{-i \omega(k-j)} d \omega \\
& =\frac{1}{2 \pi} \sum_{s=1}^{M} \int_{-\pi}^{\pi} \Phi_{w}(\omega)\left|\sum_{j=1}^{N} p_{s j} e^{-i \omega j}\right|^{2} d \omega \\
& \leq \max _{\omega} \Phi_{w}(\omega) \sum_{s=1}^{M} \frac{1}{2 \pi} \int_{-\pi}^{\pi}\left|\sum_{j=1}^{N} p_{s j} e^{-i \omega j}\right|^{2} d \omega \\
& =\max _{\omega} \Phi_{w}(\omega) \sum_{s=1}^{M} \sum_{j=1}^{N} p_{s j}^{2} \\
& =M \max _{\omega} \Phi_{w}(\omega)
\end{aligned}
$$

since $\left|p_{s}\right|=1, \forall s$. This completes the proof.

Lemma B.2 Let $x_{N}$ be defined by (62)-(63), and assume that $R_{N}>\delta I$. Assume also that

$$
\varphi(t)=[u(t), u(t-1), \ldots, u(t-M+1)]^{T}
$$

where $\{u(t)\}$ is a periodic sequence with periodicity $P \in[1, \infty)$. Then

$$
\left.x_{N}^{2} \leq \max _{\omega}|W(\omega)|^{2} \cdot \frac{(1+\log (1+N / P))^{2}}{N} \cdot \frac{M P^{2}}{\delta} \cdot \max _{1 \leq t \leq P} \mid u(t)\right)\left.\right|^{2}
$$


Here $|W(\omega)|^{2}$ is the periodogram of $w(t)$ :

$$
|W(\omega)|^{2}=\frac{1}{N}\left|\sum_{t=1}^{N} w(t) e^{-i \omega t}\right|^{2}
$$

Proof: We have

$$
\begin{aligned}
x_{N}^{2} & =\left|R_{N}^{-1 / 2} \frac{1}{N} \sum_{t=1}^{N} w(t) \varphi(t)\right|^{2} \leq\left(\delta N^{2}\right)^{-1}\left|\sum_{t=1}^{N} w(t) \varphi(t)\right|^{2} \\
& =\left(\delta N^{2}\right)^{-1}\left|\frac{1}{2 \pi} \int_{0}^{2 \pi}\left(\sum_{t=1}^{N} w(t) e^{-i \omega t}\right)\left(\sum_{s=1}^{N} \varphi(s) e^{i s \omega}\right) d \omega\right|^{2} \\
& =\left(\delta N^{2}\right)^{-1} \sum_{j=0}^{M-1}\left[\frac{1}{2 \pi} \int_{0}^{2 \pi}\left(\sum_{t=1}^{N} w(t) e^{-i \omega t}\right)\left(\sum_{s=1}^{N} u(s-j) e^{i s \omega}\right) d \omega\right]^{2} \\
& \leq(\delta N)^{-1} \cdot \max _{\omega}|W(\omega)|^{2} \cdot \sum_{j=0}^{M-1}\left(\frac{1}{2 \pi} \int_{0}^{2 \pi}\left|\sum_{s=1}^{N} u(s-j) e^{i s \omega}\right| d \omega\right)^{2}
\end{aligned}
$$

Let us consider the terms in the last factor. Write $N$ as

$$
N=P\left[\frac{N}{P}\right]+r, \quad r \in[0, P-1]
$$

where $[k]$ means the integer part of $k$. Then

$$
\begin{aligned}
& \frac{1}{2 \pi} \int_{0}^{2 \pi}\left|\sum_{s=1}^{N} u(s-j) e^{i s \omega}\right| d \omega \\
\leq & \frac{1}{2 \pi} \int_{0}^{2 \pi}\left[\sum_{s=1}^{r}\left|\sum_{t=0}^{\left[\frac{N}{P}\right]} u(P t+s-j) e^{i \omega(P t+s)}\right|+\sum_{s=r+1}^{P}\left|\sum_{t=0}^{\left[\frac{N}{P}\right]-1} u(P t+s-j) e^{i \omega(P t+s)}\right|\right] d \omega \\
= & \frac{1}{2 \pi} \int_{0}^{2 \pi}\left[\sum_{s=1}^{r}\left|\sum_{t=0}^{\left[\frac{N}{P}\right]} u(s-j) e^{i \omega(P t+s)}\right|+\sum_{s=r+1}^{P}\left|\sum_{t=0}^{\left[\frac{N}{P}\right]-1} u(s-j) e^{i \omega(P t+s)}\right|\right] d \omega \\
\leq & \frac{1}{2 \pi} \int_{0}^{2 \pi}\left[\sum_{s=1}^{r}|u(s-j)| \cdot\left|\sum_{t=0}^{\left[\frac{N}{P}\right]} e^{i \omega(P t+s)}\right|+\sum_{s=r+1}^{P}|u(s-j)| \cdot\left|\sum_{t=0}^{\left[\frac{N}{P}\right]-1} e^{i \omega(P t+s)}\right|\right] d \omega \\
= & \sum_{s=1}^{r}|u(s-j)| \tau(P,[N / P])+\sum_{s=r+1}^{P}|u(s-j)| \tau(P,[N / P]-1)
\end{aligned}
$$


Here we defined

$$
\tau(P, R)=\frac{1}{2 \pi} \int_{0}^{2 \pi}\left|\sum_{t=0}^{R} e^{i \omega(P t+s)}\right| d \omega
$$

(Note that the expression in fact is independent of $s$.) We shall soon establish that

$$
\tau(P, R) \leq 1+\log (R+1)
$$

We can now collect this bound together with the inequality above and (75) to find that

$$
x_{N}^{2} \leq(\delta N)^{-1} \cdot \max _{\omega}|W(\omega)|^{2} \cdot \sum_{j=0}^{M-1}\left(\left(1+\log \left(\left[\frac{N}{P}\right]+1\right)\right)\right)^{2}\left(\sum_{s=1}^{P}|u(s)|\right)^{2}
$$

which gives the desired result of the lemma.

It now only remains to establish (75). The integrand in the definition of $\tau$ can be summed to

$$
\kappa(\omega)=\left|\frac{1-e^{i \omega P(R+1)}}{1-e^{i \omega P}}\right|
$$

It is easy to see that the integral over this function, with periodicity $\pi / P$, will in fact be independent of $P$. Let's take $P=1$. The denominator is then equal to $2 \sin (\omega / 2)$. Jordan's inequality

$$
\sin x \geq \frac{2}{\pi} x \text { for } \omega \in[0, \pi / 2]
$$

thus gives that

$$
\kappa(\omega) \leq \frac{\pi}{\omega} \omega \in[0, \pi]
$$

We also have that the integrand is bounded by its maximal value (obtained for $\omega=0$ )

$$
\kappa(\omega) \leq R+1
$$

We now have

$$
\begin{aligned}
\frac{1}{2 \pi} \int_{-\pi}^{\pi} \kappa(\omega) d \omega & =\frac{1}{\pi} \int_{0}^{\pi} \kappa(\omega) d \omega=\frac{1}{\pi} \int_{0}^{\pi /(R+1)} \kappa(\omega) d \omega+\frac{1}{\pi} \int_{\pi /(R+1)}^{\pi} \kappa(\omega) d \omega \\
& \leq \frac{1}{\pi} \int_{0}^{\pi /(R+1)}(R+1) d \omega+\frac{1}{\pi} \int_{\pi /(R+1)}^{\pi} \frac{\pi}{\omega} d \omega=1+\log (R+1)
\end{aligned}
$$




\section{References}

[1] J.R. Deller. Set membership identification in digital signal processing. IEEE ASSP Magazine, 4:4-20, 1989.

[2] N.R. Draper and H. Smith. Applied Regression Analysis, 2nd ed. Wiley, New York, 1981.

[3] G.C. Goodwin, M. Gevers, and B. Ninness. Quantifying the error in estimated transfer functions with application to model order selection. IEEE Trans. Automatic Control, 37(7):913-929, 1992.

[4] G.C. Goodwin and M. Salgado. A stochastic embedding approach for quantifying uncertainty in estimation of restricted complexity models. Int. J. of Adaptive Control and Signal Processing, 3:333-356, 1989.

[5] L. Guo and C. Wei. Robust identification of systems with both bias and variance disturbances. Chinese Science Bulletin, 39(20):1673-1679, 1994.

[6] H. Hjalmarsson and L.Ljung. A discussion of "unknown-but-bounded" disturbances in system identification. In Proc. 32nd IEEE Conf. on Decision and Control, San Antonio, Texas, 1993.

[7] R.L. Kosut, G. C. Goodwin, and M. P. Polis (Eds). Special Issue on System Identification for Robust Control Design, IEEE Trans. Automatic Control, Vol 3\%. 1992.

[8] R.L. Kosut, M.K. Lau, and S.P. Boyd. Set-membership identification of systems with parametric and nonparametric uncertainty. IEEE Trans. Autom. Control, 37(7):929-942, 1992.

[9] L. Ljung. System Identification - Theory for the User. Prentice-Hall, Englewood Cliffs, N.J., 1987.

[10] M. Milanese and R. Tempo. Optimal algorithms for robust estimation and prediction. IEEE Trans. Automatic Control, AC-30:730-738, 1985.

[11] K. Poolla, P. P. Khargonekar, A. Tikku, J. Krause, and K.Nagpal. A time-domain approach to model validation. IEEE Trans. on Automatic Control, AC-39:951-059, 1994. 
[12] F.C. Schweppe. Uncertain Dynamical Systems. Prentice-Hall, Englewood Cliffs, 1973.

[13] R.S. Smith and J.C Doyle. Model invalidation: A connection between robust control and identification. IEEE Trans. Automatic Control, 37:942952, July 1992.

[14] T. Söderström and P. Stoica. System Identification. Prentice-Hall International, Hemel Hempstead, Hertfordshire, 1989.

[15] B. Wahlberg and L. Ljung. Hard frequency-domain model error bounds from least-squares like identification techniques. IEEE Trans. on Automatic Control, pages 900-912, 1992.

[16] E. Walter and H. Piet-Lahanier. Exact and recursive description of the feasible parameter set for bounded error models. In Proc 26 IEEE Conf. on Decision and Control, pages 1921-1922, Los Angeles, 1987.

ljung/papers/modval/modval.tex 